This item was submitted to Loughborough's Research Repository by the author.

Items in Figshare are protected by copyright, with all rights reserved, unless otherwise indicated.

\title{
Healthy obesity as an intermediate state of risk: A critical review
}

PLEASE CITE THE PUBLISHED VERSION

http://dx.doi.org/10.1080/17446651.2016.1220298

\section{PUBLISHER}

() Expert Reviews. Published by Taylor and Francis

\section{VERSION}

AM (Accepted Manuscript)

\section{PUBLISHER STATEMENT}

This work is made available according to the conditions of the Creative Commons Attribution-NonCommercialNoDerivatives 4.0 International (CC BY-NC-ND 4.0) licence. Full details of this licence are available at: https://creativecommons.org/licenses/by-nc-nd/4.0/

\section{LICENCE}

CC BY-NC-ND 4.0

\section{REPOSITORY RECORD}

Bell, Joshua A., and Mark Hamer. 2019. "Healthy Obesity as an Intermediate State of Risk: A Critical Review". figshare. https://hdl.handle.net/2134/22265. 
Healthy Obesity as an Intermediate State of Risk: A Critical Review

Word count: Summary $=189 ;$ Main text $=6145$

References: 115 


\section{Abstract}

Introduction: Obesity is a top public health priority but interventions to reverse the condition have had limited success. About 1-in-3 obese adults are free of metabolic risk factor clustering and are considered 'healthy', and much attention has focused on the implications of this state for obesity management.

Areas covered: We searched for individual studies, systematic reviews, and meta-analyses which examined correlates and outcomes of metabolically healthy obesity. We discuss the key roles of fat distribution and physical activity in determining healthy vs. unhealthy obesity and report a greatly increased risk of incident type 2 diabetes associated with healthy obesity vs. healthy normal-weight, among other outcomes. We argue that despite inconsistencies in the definition, patterns across studies clearly show that healthy obesity is a state of intermediate disease risk.

Expert commentary: Given the current state of population-level evidence, we conclude that obesity and metabolic dysfunction are inseparable and that healthy obesity is best viewed only as a state of relative health but not of absolute health. We recommend that weight loss through energy restriction be a stand-alone target in addition to increased physical activity for minimising risk of future disease.

\section{Keywords}

Obesity; Body mass index; Metabolic health; Physical activity; Cardiorespiratory fitness; Type 2 diabetes; Cardiovascular disease; Mortality; Epidemiology; Review 


\section{The stubborn issue of obesity}

The global prevalence of obesity has tripled since the mid-1970s (1), with about 1 -in-3 adults in the United States (2) and about 1-in-4 adults in the United Kingdom (3) now considered obese. These levels are concerning primarily because of the high burden of chronic disease linked with obesity; these most notably include type 2 diabetes, cardiovascular diseases, musculoskeletal disorders, depression, and several types of cancer (4), culminating in a reduced quality of life and an increased risk of early death $(5,6)$. Apart from personal strains placed on individuals and families affected, this high prevalence of obesity confers extortionate financial costs to health and social care systems, often running into the tens of billions per year (7).

Most studies measure obesity using body mass index (BMI) which is an indirect measure of body fat expressed as a simple ratio of weight in kilograms over height in meters-squared. BMI does not distinguish fat from lean mass and does not describe how fat is distributed within the body, but it nevertheless remains a validated measure against dual-X-ray absorptiometry (DXA)-assessed adiposity for both men and women $(8,9)$ and a consistent predictor of real clinical outcomes including type 2 diabetes (10), cardiovascular disease (11), and premature mortality $(5,6)$, often to the same degree as more localised fat indicators. For example, a standard unit increase in each of $\mathrm{BMI}$ and waist circumference is associated with a near-doubling in risk of developing type 2 diabetes (12).

Reversing the obesity epidemic has proven to be challenging. Given the extraordinarily wide range of proximal and distal factors which contribute to a positive energy balance $(13,14)$, it is perhaps not surprising that individual-level diet- and physical activity-based interventions aimed at tipping this balance tend initially to be moderately effective at reducing weight (15), but then tend to lose effectiveness over longer periods, resulting in weight-regain in most cases (16). The reality is that 
once established, obesity is difficult to reverse - only about 1 in 100 obese adults and about 1 in 1000 morbidly obese adults annually achieve a normal-weight (17). The stubbornness of obesity has motivated deeper investigations into what exactly makes it harmful for the sake of prioritising individual and public resources - if obesity cannot be reversed, can it at least be made harmless?

\section{A 'healthy' type of obesity}

The bulk of the disease burden associated with obesity is thought to be mediated through disruptions to metabolism (18), and so one novel way of studying obesity has been to separate one's obesity status from one's metabolic health status in order to examine whether metabolic functioning can be preserved despite being obese. Beyond this basic intention, 'healthy' obesity is a matter of definition. Most studies base their definition of health on the absence of metabolic risk factor clustering, using criteria such as that proposed by the US National Cholesterol Education Program (19) which requires no more than 2 of 6 risk factors to be present in defining health (high blood pressure, high blood glucose, high triglycerides, low HDL cholesterol, and high sex-specific waist circumference). Other criteria exclude waist circumference and additionally consider insulin resistance and systemic inflammation in a more stringent definition, requiring no more than 1 risk factor in defining health (20). Owing to high correlations between BMI and waist circumference (21), only one obesity indicator is best used at a time in such definitions of healthy obesity, given that most adults who are generally obese would also be centrally obese. When applying such criteria to representative samples of the general population a substantial proportion of obese adults, about 1in-3, are considered 'healthy' $(20,22)$. By the same method, a proportion of normal-weight adults can be considered 'unhealthy', showing a clustering of the same metabolic risk factors, although this is much less common than among obese adults (about $20 \%$ of normal-weight versus about $70 \%$ of 
obese). In general, the higher the BMI group, the higher the likelihood of being classified as metabolically unhealthy. Thus, for the purposes of this review, the term 'healthy obesity' will be used to refer to obesity that presents without metabolic dysfunction; criteria and cut-points vary by study.

\section{What explains healthy obesity?}

In asking what factors explain healthy obesity, recent research has focused on differences in underlying physiology. Studies using magnetic resonance imaging (MRI) and computerised tomography (CT) technologies have long-documented distinct typologies of fat stores and their links with metabolic functioning (23), making fat distribution a strong candidate in explaining relative health among the obese. A broad distinction is usually made between subcutaneous fat which is located peripherally in places such as arms and legs, and visceral fat which is located centrally in places such as the abdomen and around or within internal organs; the latter being more metabolically active and more concentrated with adipocytes which induce systemic inflammation (24), insulin resistance (25), and hyperglycaemia (26). A potential upstream determinant of fat distribution is the ability of subcutaneous fat cells to expand in response to a surplus of incoming triglycerides (27-29); this implies that some obese adults are better able to contain fat within relatively safe stores resulting in a healthy profile, while others are prone to having this fat 'spill over' into unsafe stores resulting in an unhealthy profile.

Several population-based studies have reported a lower waist circumference among healthy than among unhealthy obese adults $(20,30)$ despite similar levels of BMI. More detailed assessments confirm lower volumes of visceral fat among the healthy versus the unhealthy obese, despite littleto-no difference in subcutaneous fat between them (31-33). Healthy obese women have also shown 
more subcutaneous fat in the thigh than unhealthy obese women (31), likely reflecting a genderbiased pattern of fat storage. Others have gone further, demonstrating lower traces of fat in the liver among healthy than among unhealthy obese adults (34-36), which is strongly deterministic of insulin resistance (25). A recent experimental study demonstrated that adults who were initially unhealthy obese were more likely than those initially healthy obese to gain fat in visceral stores in response to an equal amount of diet-induced weight gain over a period of 2 weeks (37). This weight gain resulted in a worsened metabolic profile among the initially unhealthy obese, but not among the initially healthy obese, suggesting that a superior ability to store fat in relatively safe subcutaneous stores does indeed help to explain relative health among the obese.

Looking beyond physical characteristics, we can look to a behaviour which is robustly associated with features of metabolic risk factor clustering - physical activity (18). Whether done for the purpose of sport or tasks of daily living, physical activity of at least a moderate intensity results in the contraction of skeletal muscle which is recognised to be an active endocrine organ that in turn stimulates the release of acute pro-inflammatory and chronic anti-inflammatory cytokines which together promote a robust immune system (38). Muscle contraction also improves sensitivity of muscle tissue to insulin and facilitates the absorption of glucose into muscle, which directly enables energy expenditure and maintenance of normal glucose levels in the blood (39); contraction also releases lipoprotein-enzyme complexes which break-down triglycerides in the blood and prevent their deposition into subcutaneous or visceral fat stores $(40,41)$. Additionally, the contraction of muscle is effective in maintaining normal resting levels of blood pressure, with meta-analyses of randomised controlled trials confirming this to be true in response to either resistance- or aerobicbased physical activity $(42,43)$. When considering metabolic risk factors collectively, physical activity consistently reduces risk of their clustering among adults in large population-based samples $(44,45)$. Another key benefit of physical activity which extends beyond metabolism is for improving bodywide utilisation of oxygen by way of increased cardiorespiratory fitness (46), for which associations with physical activity are dose-response in nature (47). 
Physical activity levels tend to be higher among adults who are metabolically healthy versus unhealthy and among adults who are normal-weight versus obese $(48,49)$. Less is known about whether healthy obese adults are more physically active than their unhealthy obese counterparts. This seems intuitive, but several cross-sectional studies reported no difference in total physical activity between healthy and unhealthy obese groups $(30,33,50)$, with differences in moderate-tovigorous physical activity being evident in only half $(20,33)$ of the studies which examined this $(30$, 51). All of these studies utilised self-reported questionnaire-based measures of physical activity duration, which are known to have low-to-moderate correlations with objective accelerometerbased activity measures (52), and for which measurement error may be highest among the obese due to inaccurate recall or social desirability biases (53). When examining differences in total physical activity between healthy and unhealthy obese adults using an accelerometer, we demonstrated that total physical activity was higher among healthy versus unhealthy adults of any BMI group, including the obese, with a clear graded pattern of less physical activity among more adverse metabolic and obesity combinations (54). One additional study reported slightly higher levels of cardiorespiratory fitness among healthy than among unhealthy obese adults, by about 0.5 METs (55); this difference was apparent when using either body fat percentage or BMI to define obesity. Together, this suggests that higher physical activity and higher cardiorespiratory fitness do help explain a healthier metabolic profile among the obese; however these levels are only relatively higher, given that both physical activity and fitness are still much lower among healthy obese than among healthy normal-weight adults $(54,55)$.

\section{Is healthy obesity harmless?}


In order to determine whether healthy obesity is harmless with respect to clinical outcomes, we must shift focus away from making comparisons between healthy and unhealthy obese adults, and begin to make comparisons between healthy obese and healthy normal-weight adults (the theoretically healthiest group). 'Healthy' is a matter of binary labelling based on clinical cut-points, and subtle differences in these metabolic risk factors may exist between obese and normal-weight adults who are both given this label, which would reveal important differences in subclinical disease burden. Studies which have examined differences in continuous levels of metabolic risk factors between healthy obese and healthy normal-weight adults report that blood lipids, blood pressure, and insulin resistance are indeed more adverse among the healthy obese $(20,36,54,55)$. Levels of coronary artery calcification have also been found to be higher among healthy obese than among healthy normal-weight adults (56), providing more direct evidence of subclinical cardiovascular disease burden; calcification was not higher in one additional study (36) but this lack of difference may be due to reliance on a restrictive sub-sample of healthy obese adults who were free of hypertension and type 2 diabetes, given that differences in calcification were mediated by such factors in the larger study (56).

As mentioned, most studies consider obese adults to be healthy if they have less than 2 of 5 metabolic risk factors, thus allowing for 1 metabolic risk factor to be present at baseline. When examining prevalence of healthy obesity using a stricter definition, allowing for 0 metabolic risk factors, about 1-in-10 obese adults are considered healthy $(57,58)$, down from about 1-in-3 when using the more lenient definition $(58,59)$, indicating that most healthy obese adults have 1 metabolic risk factor to begin with (Figure 1). Studies tend to use more lenient definitions in order to follow traditional criteria for metabolic risk factor clustering, but this may also be for practical reasons as it affords a larger group and thus greater statistical power to associate this status with clinical outcomes; relying on a strict definition leaves very few healthy obese adults left to study. 
Next, in order to determine whether healthy obesity is harmless, we must ask whether it is actually stable over time or whether it is a phase leading to unhealthy obesity. Through use of repeated measures of metabolic and obesity statuses on the same individuals over time, studies have shown that after 6 years of follow-up, about one-third of initially healthy obese adults had become unhealthy obese $(30,60)$; others report that after $7-10$ years this proportion is nearly half $(58,61$ $63)$; and after 20 years this figure is just over half (59). The proportion of healthy obese adults who progress to unhealthy obesity appears larger with longer follow-up periods, indicating that the tendency to progress gets stronger with time. When we compared tendencies for progression, adults who began as healthy obese were found to be nearly 8-times more likely to end-up as unhealthy obese after 20 years than those who began as healthy but were of a lower BMI, further positioning healthy obesity as a phase (59). This differential tendency for progression is illustrated in Figure 2. Furthermore, when using a strict definition of healthy obesity based 0 metabolic risk factors in a separate study (57), nearly $80 \%$ of these strictly healthy obese adults had developed at least 1 metabolic risk factor after 20 years (compared with $60 \%$ of strictly healthy normal-weight adults) indicating that a strictly healthy type of obesity is rarely maintained. The development of insulin resistance was largely responsible for declining metabolic health status among these strictly healthy obese adults throughout the follow-up period.

We now turn attention to real clinical outcomes. Given strong links between obesity and incident type 2 diabetes (12), numerous studies have examined whether risk is also elevated for obese adults who are metabolically healthy. We summarised results of 8 studies in a meta-analysis (64), finding that healthy obese adults have about 4-times greater risk of incident type 2 diabetes compared with healthy normal-weight adults; excess risk being highest among the unhealthy obese at nearly 9times greater risk. Some heterogeneity in effect estimates for healthy obesity was observed across the 8 studies, but this referred only to the size of effect, not the directionality of effect. Effects also did not significantly differ by duration of follow-up, indicating that high excess risk for type 2 diabetes is evident even over short periods of time. The magnitude and consistency of excess risk for 
type 2 diabetes among healthy obese adults fits with what is known about progressions from healthy to unhealthy obesity, given strong links with future insulin resistance (57) which subsequently induces hyperglycaemia. Since this meta-analysis, at least 6 additional studies have examined type 2 diabetes risk among healthy obese versus healthy normal-weight adults, all of which report excess risk of a similar magnitude (65-70).

Future risk for developing cardiovascular disease has also been widely compared between healthy obese and healthy normal-weight adults; to date, 2 meta-analyses have been published. The first of these, by Fan et al. (71), examined incident coronary heart disease or stroke across 8 studies and summarised risk of these events to be 1.5-times higher among healthy obese than among healthy normal-weight adults, this being greatest among unhealthy obese adults at about 2.9-times higher. Excess risk was higher within the 3 studies which used a follow-up duration greater than 15 years, at about 2-times higher risk, and lower but still significantly elevated within the 6 studies which used follow-up durations of 15 years or less, at about 1.4-times higher risk, suggesting that risk of developing cardiovascular disease among healthy obese adults gets higher with time. Patterns of results were expectedly similar in the second meta-analysis by Kramer et al. (72), albeit with less clarity over the outcome being examined, given that incident cardiovascular events were grouped together with all-cause and cardiovascular-related mortality as a single composite outcome. Still, the 4 studies which used follow-up durations of at least 10 years showed about 1.2-times higher risk among healthy obese than among healthy normal-weight adults, and the events being examined in these 4 studies were in fact incident cardiovascular events, not mortality (73-76). Once again, excess risk among unhealthy obese adults was greatest, at about 2.6-times higher.

Most studies to date have focused on the metabolic or cardiovascular outcomes of healthy obesity, but these are not the only important domains of health. For instance, given obesity's strong link with development of kidney disease (6), several recent studies have examined whether this is also true of healthy obese adults. The risk for both incident kidney disease $(77,78)$ and of end-stage kidney 
disease is reported to be higher among healthy obese than among healthy normal-weight adults (79) across 3 studies, excess risk again being greatest among unhealthy obese adults in line with patterns observed for type 2 diabetes and cardiovascular outcomes. Given established, albeit complicated, links between BMI and development of several types of common cancer $(80,81)$, it is also worth comparing these risks between healthy obese and healthy normal-weight adults; this has not been done, but a higher risk of cancer-related mortality among healthy obese than among healthy normal-weight adults has been reported in one study (55). Additionally, given the increased mechanical strain placed on joints by excess fat and strong links between higher BMI and development of osteoarthritis (11), a higher burden of musculoskeletal disorders may also be expected among obese adults who are metabolically healthy, but again, this has not yet been examined. We have compared prevalence of depressive symptoms between healthy obese and healthy normal-weight adults by pooling results of 8 studies in a meta-analysis (82), finding healthy obese adults to have about 1.3-times higher risk for being depressed than healthy normal-weight adults; excess risk again being highest among unhealthy obese adults at about 1.6-times higher. Finally, looking beyond the development of disease to the risk of early death, a meta-analysis (71) reviewed 9 studies and summarised risk for death from all causes to be about 1.2-times higher among healthy obese than among healthy normal-weight adults. Excess risk of death due specifically to cardiovascular causes was nearly 1.4-times higher among the healthy obese based on 6 studies. As mentioned, healthy obese adults have also shown excess risk for death due to cancer, although based on a single study (55).

Thus, when considering prospective evidence linking healthy obesity with a range of clinical outcomes, it becomes clear that healthy obesity is not really healthy in an absolute sense. Healthy obese adults tend to begin prospective studies with more adverse levels of individual metabolic risk factors than healthy normal-weight adults, and they are also far more likely to progress to unhealthy obesity. Healthy obese adults are consistently more likely than healthy normal-weight adults to develop a range of chronic diseases, the strongest and most consistent links being with development 
of type 2 diabetes, supporting inseparable links between excess body fat and poor glucose control. These trends culminate in an increased risk of early death related to cardiovascular- and cancerrelated causes. For each outcome studied, it is also clear that healthy obesity is an intermediate state of risk; excess risk for disease is not as high as for unhealthy obesity, but is still higher than for healthy normal-weight. These patterns are illustrated in Figure 3. Indeed, despite widely varying definitions of healthy obesity used across studies, the patterns for incident disease are remarkably consistent; effect sizes do vary, but conclusions do not.

\section{What explains declining health among the initially healthy obese?}

Having observed strong tendencies for declining health and for future disease among initially healthy obese adults, it is worth addressing factors which may influence these outcomes. Studies typically account for a range of demographic and health-related factors when estimating risk, but a review of these factors may be best focused on fat distribution and physical activity given their strong links with metabolic health. How we address such factors depends on what role they are theorised to play in associations between healthy obesity and decline - whether as confounders, as mediators, or as effect modifiers (83).

When examining the role of fat distribution in progressions from healthy to unhealthy obesity, at least 4 studies have reported that stability of a healthy obese status over time is linked with a lower waist circumference relative to healthy obese adults who progressed to ill-health $(30,58,61,62)$, indicating that maintaining a favourable fat distribution may protect against decline. One additional study reported that excess risk for type 2 diabetes among healthy obese adults was evident only among those who also had traces of fat in the liver (69), suggesting that fat distribution may modify type 2 diabetes risk. Muscle mass may also modify type 2 diabetes risk given insight from studies on 
dynapenic obesity among older adults; these tend to show that obesity in combination with a low grip-strength confers greater risk for type 2 diabetes than either obesity or low grip-strength on its own (84), possibly due to effects of insulin resistance resulting from low muscle mass (85). Furthermore, 2 studies have shown that excess type 2 diabetes risk is not evident among healthy obese adults who maintain their healthy state over the duration of follow-up $(30,86)$; declining health status likely being an effect modifier due to its strong mediating role for disease development, with progressions to ill-health being an inherent part of the disease process.

Adjustment for initial levels of physical activity in 2 studies examining progressions from healthy to unhealthy obesity found no evidence for confounding by physical activity $(30,58)$, while 1 study did (61), albeit while not separating obese adults from overweight adults in the comparison group. Adjustment for physical activity was made in 4 out of the 8 studies $(64,87-89)$ included in the metaanalysis of incident type 2 diabetes (64), and this had minimal impact on effect estimates, with excess risk among healthy obese adults remaining significantly elevated in each study. Thus, excess risk for type 2 diabetes among healthy obese adults does not appear to be explained by differential engagement in physical activity. Results are less clear in relation to cardiovascular disease. Adjustment for physical activity was made in only 2 out of 8 studies $(76,90)$ included in a metaanalysis of incident cardiovascular disease (71), but excess cardiovascular risk among healthy obese adults was absent before adjustment in both studies. Adjustment for cardiorespiratory fitness did attenuate associations between healthy obesity and incident cardiovascular events in one study (55); the same was apparent for cardiovascular-related mortality. Excess risk for cancer-related mortality among healthy obese adults remained evident after adjustment for fitness in the same study.

When studies adjust for physical activity or fitness they assume that either factor acts as a simple confounder, such that differences in risk of the outcome between healthy obese and healthy normal-weight adults either partially or entirely reflect differences in physical activity or fitness. 
They also assume that effects of physical activity or fitness are equal among exposure groups. If, however, there is evidence that physical activity mediates associations between healthy obesity and metabolic decline then it would not be appropriate to adjust for physical activity as it would lie on the causal pathway. Several prospective observational studies suggest that higher adiposity leads to inactivity and not vice versa $(91,92)$, and results from a recent study among children which examined genetically elevated BMI in relation to objectively measured physical activity using a Mendelian randomisation approach found evidence for a causal association between higher BMI and lower subsequent physical activity (93). This study is particularly valuable as it avoids issues of confounding and reverse causality which are inherent to observational epidemiology, given that robustly associated genetic variants which are assigned randomly and independently at conception are used as proxies for measured BMI $(94,95)$. Nevertheless, pleiotropic influences cannot be discounted.

Whether physical activity or fitness modifies excess disease risk among healthy obese adults has not been specifically examined, however these effects may be inferred from previous work comparing risk among highly active or highly fit obese adults with similarly active or fit normal-weight adults, assuming that there are considerable overlaps with metabolic health statuses. In 2 such studies, obese adults who were highly active showed over 10-times greater risk for incident type 2 diabetes than normal-weight adults who were similarly active $(96,97)$; another study reported that obese adults who were highly fit carried 3-times higher risk for incident type 2 diabetes than normalweight adults who were also highly fit (98). A systematic review later confirmed these trends for impaired glucose control and type 2 diabetes, additionally concluding that excess risk among highly active or fit obese adults is even higher than among normal-weight adults who are inactive or unfit (99). Patterns of risk appear similar for cardiovascular outcomes, with physically active obese adults showing higher risk for cardiovascular events than physically active normal-weight adults in 4 studies (100-103), this excess risk being nearly 3-times higher in half of these $(102,103)$. 
A single baseline measure of physical activity is not likely sufficient to truly stratify risk groups, given that this behaviour is subject to considerable change over the duration of follow-up. Ideally, incident disease risk would be examined separately among healthy obese adults who maintain high levels of physical activity over the duration of follow-up and among those who do not maintain high levels based on repeated activity measures, but this is often not feasible given that such repeated measures may not be available and that few healthy obese individuals would exist in each stratified group, reducing the statistical power needed to detect meaningful differences. In such cases where high physical activity is maintained, 'obesity' likely refers to a high muscle and subcutaneous fat mass with minimal visceral fat (104). Randomised controlled trials using strictly supervised physical activity regimes have shown these to be effective at reducing abdominal and visceral fat even in the absence of total weight loss (104-106); these reductions improve glucose control and may in turn minimise disease risks. However, given other studies reviewed which suggest that physical activity does not eliminate excess risk for eventual disease among obese adults, a key issue here is likely the maintenance of such physical activity over time which is notoriously low among obese adults (107). Physical activity is only expected to be protective for as long as it is maintained and if maintenance is less likely among obese than among normal-weight adults then even an obesity paired with high physical activity would still be considered a high risk state for future decline. An important but unanswered question is once habitual physical activity ceases, whether the likelihood of progressing to metabolic ill-health is greater the higher the underlying BMI.

Observations that some excess risk for disease remains among obese adults who are metabolically healthy or who are physically active or fit suggest that there are inherent links between excess body fat and metabolic dysfunction. Such links are supported by at least 2 large Mendelian randomisation studies which recently examined causal effects of genetically elevated BMI on a range of metabolic risk factors, many of which are commonly used to define health among the obese. One of these studies, by Holmes et al. (108), reported causal effects of genetically elevated BMI on higher levels of fasting glucose, insulin, systemic inflammation, and blood pressure, with mixed results for blood 
lipids. Genetically elevated BMI also showed a clear causal effect on type 2 diabetes development, at about 1.3-times higher risk per unit increase in the BMI genetic risk score; this was not evident for coronary heart disease or stroke (108). A second study, by Würtz et al. (109), reported adverse causal effects of genetically elevated BMI on blood-based metabolites of lipoproteins, lipids, fatty acids, and glucose among others, based on a large sample of young adults whose BMI was below the obese range. Effect sizes were larger among those who had gained weight over 6 years (109). Thus, the main reason why obese adults who are relatively healthy or physically active still tend to experience metabolic decline, is likely that obesity is itself causing this decline. Obesity and metabolic dysfunction are truly inseparable.

\section{Does weight loss benefit healthy obese adults?}

Given known excess risk for a range of diseases among healthy obese adults, it follows that weight loss should be recommended in order to reduce these risks; but is there evidence that this works? When considering all obese adults collectively, significant metabolic benefits are known to occur with as little as $5 \%$ weight loss, with the magnitude of these benefits being progressively greater for weight loss of $10 \%$ and $15 \%$ (110). Several studies have compared these benefits between obese adults who were initially healthy versus those who were initially unhealthy, and at face value, results appear to be mixed. For example, in response to an intervention based entirely on energy restriction, one 12-week study reported improvements to systemic inflammation and to a range of blood lipids among initially unhealthy obese but not among initially healthy obese adults (111); insulin resistance decreased in both groups although not at a significant level. Another 6-month energy restriction-based intervention reported improved insulin sensitivity among the initially 
unhealthy obese, but worsened insulin sensitivity among the initially healthy obese (112), albeit while not measuring other metabolic risk factors.

Interventions which involve physical activity tend to report more positive results. One 6-month study involving an intervention based only on physical activity reported that initially healthy obese adults reduced insulin secretion and blood glucose concentrations while also increasing cardiorespiratory fitness relative to similarly healthy obese controls (113), this was in addition to reduced total body weight, BMI, and waist circumference. Two additional studies reported on interventions which combined energy restriction with physical activity, the first of which spanned 6 months and found improved insulin sensitivity among both initially unhealthy and initially healthy obese adults in addition to reduced total, subcutaneous, and visceral fat mass, and preserved muscle mass (114). The second 9-month intervention reported reductions in total and visceral fat mass in both groups but increased insulin sensitivity among obese adults who were initially unhealthy only (115).

Greater improvements in metabolic risk factors would be expected among those obese adults who began as unhealthy, given that they had more room to improve. Unhealthy obese adults are also expected to begin interventions with higher volumes of visceral fat than the healthy obese, higher levels indeed being reported in studies which measured this at baseline $(111,114,115)$. Given that healthy obese adults have less potential to increase insulin sensitivity by way of reducing visceral fat, mechanisms related to physical activity may be more effective. Clear benefits to body composition are observed for both initially healthy and unhealthy obese adults in response to interventions which involve physical activity as these can preserve or increase muscle mass and stimulate muscle contraction, which together can result in improved insulin sensitivity and a greatly reduced risk for future type 2 diabetes. However, the greatest long-term benefits may still be expected with increased physical activity in combination with energy restriction to reduce BMI to at least below the obese range, as such high BMI may be a barrier to maintaining physical activity over time. 


\section{Concluding remarks}

The idea of a 'healthy' type of obesity is based on the observation that a sizable minority of obese adults, about 1-in-3, present without a clustering of metabolic risk factors. This state is likely explained through a favourable fat distribution by way of higher volumes of subcutaneous relative to visceral fat, and a favourable behavioural profile by way of higher physical activity, among other factors. When examining how these individuals change over time, however, prospective evidence has revealed that 'healthy obesity' is a highly misleading term as this state very often progresses into unhealthy obesity through incidence of metabolic risk factors, and very often results in the development of several types of disease. Excess risk is strongest and most consistent for development of type 2 diabetes, supporting inseparable links between obesity and poor glucose control. Other evidence suggests that excess risk for type 2 diabetes among obese adults remains elevated even among those who are physically active or fit, although these factors may reduce the magnitude of excess risk. These patterns of disease risk are remarkably consistent despite widely varying definitions of healthy obesity used in literature. For virtually every disease outcome examined healthy obesity is merely a state of intermediate risk; excess risk is not as high as for unhealthy obesity, but it is still higher than for healthy normal-weight. Healthy obesity is therefore best viewed only as a state of relative health but not of absolute health; it is better than unhealthy obesity but it is still not safe or harmless. Weight loss through energy restriction should therefore be a stand-alone target in addition to increased physical activity for minimising risk of future disease.

\section{Expert commentary}


Obesity is very difficult to reverse. In light of this, the idea that obesity can exist without conferring metabolic dysfunction is highly attractive as this implies that these individuals are protected against future disease. Recent evidence has all but dismissed this idea. Although it is true that a sizable minority of obese adults are free of metabolic risk factor clustering and are considered 'healthy', when followed-up over time, these individuals demonstrate a strong tendency to progress to unhealthy obesity and to develop numerous chronic diseases including type 2 diabetes and cardiovascular disease. These outcomes occur at rates greater than among normal-weight adults who are similarly healthy and are not entirely explained by a lack of physical activity. New studies which utilise common genetic variants as proxies for body mass index have suggested that it is obesity itself which causes harm, and such evidence will likely continue to improve knowledge of causal links between obesity, low physical activity, and metabolic dysfunction in the future. Overall, the current state of population-level evidence strongly suggests that obesity and metabolic dysfunction are inseparable and that so-called healthy obesity is healthy only in a relative sense but not an absolute sense. The recommendation for public health is therefore to set weight loss through energy restriction as a stand-alone target in addition to regular physical activity for minimising risk of future disease.

\section{Five year view}

The topic of healthy obesity has progressed immensely in a short time. Five years ago, the recognition of a metabolically healthy type of obesity was thought to represent a paradigm shift in disease aetiology with obesity viewed as a separate entity from metabolic ill-health; these entities are now viewed as inseparable. This shift in understanding came from prospective evidence on how healthy obese adults change over time and how their risk of future disease differs from similarly 
healthy normal-weight adults. Evidence on causal links between genetically elevated body mass index and subsequent low physical activity and metabolic dysfunction has also been valuable. In the next five years, such prospective and genetically informed evidence will likely continue to provide insight into disease outcomes of obesity which are beyond the traditional cardio-metabolic domain in order to better quantify obesity's total impact on quality of life. Researchers and public health practitioners will likely gain a greater appreciation for the continuous nature of chronic disease risk which is better represented by a spectrum than by clinical cut-points. Long-term randomised controlled trials on the combined protective effects of maintaining high physical activity and a low energy/high nutrient diet from minimally processed food sources on risk of future disease among adults of different body mass indexes will be informative, as will be studies using genetic variants as proxies for risk factors in cases where such trials are not feasible. Lastly and importantly, it is hoped that a focus on the main systemic driver of weight gain in populations will be intensified through government action on improving the quality of the food supply.

\section{Key issues}

- At any given time, about 1-in-3 obese adults are free of metabolic risk factor clustering and are considered 'healthy'. This healthy obese state is likely induced through lower visceral relative to subcutaneous fat and through higher physical activity relative to the unhealthy obese state.

- Despite both groups falling below clinical cut-points for defining 'metabolic ill-health', healthy obese adults show more adverse levels of metabolic risk factors than similarly healthy normalweight adults. Healthy obese adults are also far more likely than healthy non-obese adults to progress to unhealthy obesity over time; this is likely driven by insulin resistance. 
- Compared with healthy normal-weight adults, healthy obese adults are about 4-times more likely to develop type 2 diabetes, 1.2-times more likely to develop cardiovascular disease, and 1.4-times more likely to die from cardiovascular-related causes. Excess risk is also evident for other outcomes including chronic kidney disease and death from cancer.

- Low physical activity does not fully explain excess risk for type 2 diabetes among healthy obese versus healthy normal-weight adults, with excess risk remaining elevated among physically active obese adults versus similarly active normal-weight adults. Together with known causal associations of genetically elevated body mass index on low physical activity and metabolic risk factors, this strongly suggests that obesity and metabolic dysfunction are inseparable.

- Overall, despite inconsistencies in the definition, consistent patterns across studies for a range of clinical outcomes reveal that healthy obesity is a state of intermediate risk; excess risk for disease is not as high as for unhealthy obesity but is still higher than for healthy normal-weight. Healthy obesity is therefore only a state of relative health, not absolute health.

- Weight loss through energy restriction should be a stand-alone target in addition to increased physical activity for minimising risk of future disease. 


\section{References}

1. Collaboration NRF. Trends in adult body-mass index in 200 countries from 1975 to 2014: a pooled analysis of 1698 population-based measurement studies with $19 \cdot 2$ million participants. The Lancet. 2016;387(10026):1377-96.

2. Ogden CL, Carroll MD, Kit BK, Flegal KM. Prevalence of childhood and adult obesity in the United States, 2011-2012. JAMA. 2014;311(8):806-14.

3. Scantlebury R, Moody, A. Health Survey for England 2014. Chapter 9: Adult Obesity and Overweight. The Health and Social Care Information Centre: 2015.

4. Kopelman P. Health risks associated with overweight and obesity. Obesity Reviews. 2007;8(s1):13-7.

5. Berrington de Gonzalez A, Hartge P, Cerhan JR, Flint AJ, Hannan L, Maclnnis RJ, et al. Bodymass index and mortality among 1.46 million white adults. New England Journal of Medicine. 2010;363(23):2211-9.

6. Collaboration PS. Body-mass index and cause-specific mortality in 900000 adults: collaborative analyses of 57 prospective studies. The Lancet. 2009;373(9669):1083-96.

7. Wang YC, McPherson K, Marsh T, Gortmaker SL, Brown M. Health and economic burden of the projected obesity trends in the USA and the UK. The Lancet. 2011;378(9793):815-25.

8. Blew RM, Sardinha LB, Milliken LA, Teixeira PJ, Going SB, Ferreira DL, et al. Assessing the validity of body mass index standards in early postmenopausal women. Obesity research. 2002;10(8):799-808.

9. Evans $\mathrm{E}$, Rowe D, Racette $\mathrm{S}$, Ross $\mathrm{K}$, McAuley $\mathrm{E}$. Is the current BMI obesity classification appropriate for black and white postmenopausal women? Int J Obesity. 2006;30(5):837-43.

10. Brunner EJ, Hemingway H, Walker BR, Page M, Clarke P, Juneja M, et al. Adrenocortical, autonomic, and inflammatory causes of the metabolic syndrome: nested case-control study. Circulation. 2002;106(21):2659-65. Epub 2002/11/20.

11. Guh DP, Zhang W, Bansback N, Amarsi Z, Birmingham CL, Anis AH. The incidence of comorbidities related to obesity and overweight: a systematic review and meta-analysis. Bmc Public Health. 2009;9(1):1.

12. Vazquez G, Duval S, Jacobs DR, Silventoinen K. Comparison of body mass index, waist circumference, and waist/hip ratio in predicting incident diabetes: a meta-analysis. Epidemiologic reviews. 2007;29(1):115-28.

13. Butland B, Jebb S, Kopelman P, McPherson K, Thomas S, Mardell J, et al. Foresight. Tackling obesities: future choices. Project report. Foresight Tackling obesities: future choices Project report. 2007.

14. Swinburn BA, Sacks G, Hall KD, McPherson K, Finegood DT, Moodie ML, et al. The global obesity pandemic: shaped by global drivers and local environments. The Lancet.

2011;378(9793):804-14.

15. Curioni C, Lourenco P. Long-term weight loss after diet and exercise: a systematic review. Int J Obesity. 2005;29(10):1168-74.

16. Anderson JW, Konz EC, Frederich RC, Wood CL. Long-term weight-loss maintenance: a metaanalysis of US studies. The American journal of clinical nutrition. 2001;74(5):579-84.

17. Fildes A, Charlton J, Rudisill C, Littlejohns P, Prevost AT, Gulliford MC. Probability of an obese person attaining normal body weight: cohort study using electronic health records. American journal of public health. 2015;105(9):e54-e9.

18. Frayn K. Metabolic Regulation: A Human Perspective Wiley-Blackwell; 2010.

19. Expert Panel on Detection E. Executive summary of the Third Report of the National Cholesterol Education Program (NCEP) expert panel on detection, evaluation, and treatment of high blood cholesterol in adults (Adult Treatment Panel III). JAMA. 2001;285(19):2486.

20. Wildman RP, Muntner P, Reynolds K, McGinn AP, Rajpathak S, Wylie-Rosett J, et al. The obese without cardiometabolic risk factor clustering and the normal weight with cardiometabolic 
risk factor clustering - Prevalence and correlates of 2 phenotypes among the US population (NHANES 1999-2004). Arch Intern Med. 2008;168(15):1617-24.

21. Flegal KM, Shepherd JA, Looker AC, Graubard BI, Borrud LG, Ogden CL, et al. Comparisons of percentage body fat, body mass index, waist circumference, and waist-stature ratio in adults. The American journal of clinical nutrition. 2009;89(2):500-8.

22. lacobellis G, Ribaudo MC, Zappaterreno A, lannucci CV, Leonetti F. Prevalence of uncomplicated obesity in an Italian obese population. Obesity research. 2005;13(6):1116-22.

23. Després J-P, Lemieux I, Bergeron J, Pibarot $P$, Mathieu $P$, Larose $E$, et al. Abdominal obesity and the metabolic syndrome: contribution to global cardiometabolic risk. Arteriosclerosis, thrombosis, and vascular biology. 2008;28(6):1039-49.

24. Mathieu P, Poirier $P$, Pibarot $P$, Lemieux I, Després J-P. Visceral obesity the link among inflammation, hypertension, and cardiovascular disease. Hypertension. 2009;53(4):577-84.

25. Tchernof A, Després J-P. Pathophysiology of human visceral obesity: an update. Physiological reviews. 2013;93(1):359-404.

26. Philipsen A, Jørgensen ME, Vistisen D, Sandbaek A, Almdal TP, Christiansen JS, et al. Associations between ultrasound measures of abdominal fat distribution and indices of glucose metabolism in a population at high risk of type 2 diabetes: the ADDITION-PRO study. Plos One. 2015;10(4):e0123062.

27. Sattar N, Gill JM. Type 2 diabetes as a disease of ectopic fat? BMC medicine. 2014;12(1):1.

28. Alligier M, Meugnier E, Debard C, Lambert-Porcheron S, Chanseaume E, Sothier M, et al. Subcutaneous adipose tissue remodeling during the initial phase of weight gain induced by overfeeding in humans. The Journal of Clinical Endocrinology \& Metabolism. 2011;97(2):E183-E92.

29. Alligier M, Gabert L, Meugnier E, Lambert-Porcheron S, Chanseaume E, Pilleul F, et al. Visceral fat accumulation during lipid overfeeding is related to subcutaneous adipose tissue characteristics in healthy men. The Journal of Clinical Endocrinology \& Metabolism. 2013;98(2):80210.

30. Appleton SL, Seaborn CJ, Visvanathan R, Hill CL, Gill TK, Taylor AW, et al. Diabetes and Cardiovascular Disease Outcomes in the Metabolically Healthy Obese Phenotype A cohort study. Diabetes Care. 2013.

31. Koster A, Stenholm S, Alley DE, Kim LJ, Simonsick EM, Kanaya AM, et al. Body Fat Distribution and Inflammation Among Obese Older Adults With and Without Metabolic Syndrome. Obesity. 2010;18(12):2354-61.

32. Brochu M, Tchernof A, Dionne IJ, Sites CK, Eltabbakh GH, Sims EAH, et al. What are the physical characteristics associated with a normal metabolic profile despite a high level of obesity in postmenopausal women? J Clin Endocr Metab. 2001;86(3):1020-5.

33. Hayes L, Pearce MS, Firbank MJ, Walker M, Taylor R, Unwin NC. Do obese but metabolically normal women differ in intra-abdominal fat and physical activity levels from those with the expected metabolic abnormalities? A cross-sectional study. Bmc Public Health. 2010;10.

34. Stefan N, Kantartzis K, Machann J, Schick F, Thamer C, Rittig K, et al. Identification and characterization of metabolically benign obesity in humans. Arch Intern Med. 2008;168(15):1609-16.

35. Stefan N, Häring H-U, Hu FB, Schulze MB. Metabolically healthy obesity: epidemiology, mechanisms, and clinical implications. The Lancet Diabetes \& Endocrinology. 2013;1(2):152-62.

36. Sung K-C, Cha S-C, Sung J-W, So M-S, Byrne CD. Metabolically healthy obese subjects are at risk of fatty liver but not of pre-clinical atherosclerosis. Nutrition, Metabolism and Cardiovascular Diseases. 2014;24(3):256-62.

37. Fabbrini E, Yoshino J, Yoshino M, Magkos F, Luecking CT, Samovski D, et al. Metabolically normal obese people are protected from adverse effects following weight gain. The Journal of clinical investigation. 2015;125(2):787.

38. Pedersen BK, Febbraio MA. Muscles, exercise and obesity: skeletal muscle as a secretory organ. Nature Reviews Endocrinology. 2012;8(8):457-65. 
39. Zierath JR. Invited review: exercise training-induced changes in insulin signaling in skeletal muscle. Journal of Applied Physiology. 2002;93(2):773-81.

40. Hamilton MT, Hamilton DG, Zderic TW. Role of low energy expenditure and sitting in obesity, metabolic syndrome, type 2 diabetes, and cardiovascular disease. Diabetes. 2007;56(11):2655-67.

41. Gill JM. Exercise and postprandial lipid metabolism-an analysis of the current evidence. European journal of lipid science and technology. 2004;106(2):110-21.

42. Cornelissen VA, Smart NA. Exercise training for blood pressure: a systematic review and meta-analysis. Journal of the American Heart Association. 2013;2(1):e004473.

43. Cornelissen VA, Buys R, Smart NA. Endurance exercise beneficially affects ambulatory blood pressure: a systematic review and meta-analysis. Journal of hypertension. 2013;31(4):639-48.

44. Laaksonen DE, Lakka H-M, Salonen JT, Niskanen LK, Rauramaa R, Lakka TA. Low levels of leisure-time physical activity and cardiorespiratory fitness predict development of the metabolic syndrome. Diabetes Care. 2002;25(9):1612-8.

45. Lakka TA, Laaksonen DE. Physical activity in prevention and treatment of the metabolic syndrome. Applied Physiology, Nutrition, and Metabolism. 2007;32(1):76-88.

46. Caspersen CJ, Powell KE, Christenson GM. Physical activity, exercise, and physical fitness: definitions and distinctions for health-related research. Public health reports. 1985;100(2):126.

47. Church TS, Earnest CP, Skinner JS, Blair SN. Effects of different doses of physical activity on cardiorespiratory fitness among sedentary, overweight or obese postmenopausal women with elevated blood pressure: a randomized controlled trial. JAMA. 2007;297(19):2081-91.

48. Warburton DE, Nicol CW, Bredin SS. Health benefits of physical activity: the evidence. Can Med Assoc J. 2006;174(6):801-9.

49. Sattelmair J, Pertman J, Ding EL, Kohl HW, Haskell W, Lee I-M. Dose response between physical activity and risk of coronary heart disease a meta-analysis. Circulation. 2011;124(7):789-95. 50. Phillips CM, Dillon C, Harrington JM, McCarthy VJ, Kearney PM, Fitzgerald AP, et al. Defining metabolically healthy obesity: role of dietary and lifestyle factors. Plos One. 2013;8(10):e76188.

51. Hankinson AL, Daviglus ML, Van Horn L, Chan Q, Brown I, Holmes E, et al. Diet composition and activity level of at risk and metabolically healthy obese American adults. Obesity. 2012.

52. Sabia S, van Hees VT, Shipley MJ, Trenell MI, Hagger-Johnson G, Elbaz A, et al. Association between questionnaire-and accelerometer-assessed physical activity: the role of sociodemographic factors. American journal of epidemiology. 2014;179(6):781-90.

53. Warner ET, Wolin KY, Duncan DT, Heil DP, Askew S, Bennett GG. Differential accuracy of physical activity self-report by weight status. American journal of health behavior. 2012;36(2):168. 54. Bell JA, Hamer M, van Hees VT, Singh-Manoux A, Kivimäki M, Sabia S. Healthy obesity and objective physical activity. The American journal of clinical nutrition. 2015;102(2):268-75.

55. Ortega FB, Lee DC, Katzmarzyk PT, Ruiz JR, Sui X, Church TS, et al. The intriguing metabolically healthy but obese phenotype: cardiovascular prognosis and role of fitness. European heart journal. 2012. Epub 2012/09/06.

56. Chang Y, Kim B-K, Yun KE, Cho J, Zhang Y, Rampal S, et al. Metabolically-healthy obesity and coronary artery calcification. J Am Coll Cardiol. 2014;63(24):2679-86.

57. Bell JA, Hamer M, Batty GD, Singh-Manoux A, Sabia S, Kivimäki M. Incidence of metabolic risk factors among healthy obese adults: 20-year follow-up. J Am Coll Cardiol. 2015;66(7):871-3.

58. Hamer M, Bell JA, Sabia S, Batty GD, Kivimäki M. Stability of metabolically healthy obesity over 8 years: the English Longitudinal Study of Ageing. European Journal of Endocrinology. 2015;173(5):703-8.

59. Bell JA, Hamer M, Sabia S, Singh-Manoux A, Batty GD, Kivimaki M. The natural course of healthy obesity over 20 years. J Am Coll Cardiol. 2015;65(1):101-2.

60. Soriguer F, Gutiérrez-Repiso C, Rubio-Martín E, García-Fuentes E, Almaraz MC, Colomo N, et al. Metabolically healthy but obese, a matter of time? Findings from the prospective Pizarra study. J Clin Endocr Metab. 2013. 
61. Khan UI, Wang D, Karvonen-Gutierrez CA, Khalil N, Ylitalo KR, Santoro N. Progression from metabolically benign to at-risk obesity in perimenopausal women: a longitudinal analysis of study of women across the nation (SWAN). The Journal of Clinical Endocrinology \& Metabolism.

2014;99(7):2516-25.

62. Achilike I, Hazuda H, Fowler S, Aung K, Lorenzo C. Predicting the development of the metabolically healthy obese phenotype. Int J Obesity. 2015;39(2):228-34.

63. Eshtiaghi R, Keihani S, Hosseinpanah F, Barzin M, Azizi F. Natural course of metabolically healthy abdominal obese adults after 10 years of follow-up: the Tehran Lipid and Glucose Study. Int J Obesity. 2015;39(3):514-9.

64. Bell J, Kivimaki M, Hamer M. Metabolically healthy obesity and risk of incident type 2 diabetes: a meta-analysis of prospective cohort studies. Obesity Reviews. 2014;15(6):504-15. 65. Hinnouho G-M, Czernichow S, Dugravot A, Nabi H, Brunner EJ, Kivimaki M, et al. Metabolically healthy obesity and the risk of cardiovascular disease and type 2 diabetes: the Whitehall II cohort study. European heart journal. 2015;36(9):551-9.

66. Aung K, Lorenzo C, Hinojosa MA, Haffner SM. Risk of developing diabetes and cardiovascular disease in metabolically unhealthy normal-weight and metabolically healthy obese individuals. The Journal of Clinical Endocrinology \& Metabolism. 2013;99(2):462-8.

67. Heianza Y, Kato K, Kodama S, Ohara N, Suzuki A, Tanaka S, et al. Risk of the development of Type 2 diabetes in relation to overall obesity, abdominal obesity and the clustering of metabolic abnormalities in Japanese individuals: does metabolically healthy overweight really exist? The Niigata Wellness Study. Diabetic Medicine. 2015;32(5):665-72.

68. Twig G, Afek A, Derazne E, Tzur D, Cukierman-Yaffe T, Gerstein HC, et al. Diabetes risk among overweight and obese metabolically healthy young adults. Diabetes Care. 2014;37(11):2989-95.

69. Heianza Y, Arase Y, Tsuji H, Fujihara K, Saito K, Hsieh SD, et al. Metabolically healthy obesity, presence or absence of fatty liver, and risk of type 2 diabetes in Japanese individuals: Toranomon Hospital Health Management Center Study 20 (TOPICS 20). The Journal of Clinical Endocrinology \& Metabolism. 2014;99(8):2952-60.

70. Jung $\mathrm{CH}$, Lee MJ, Kang YM, Jang JE, Leem J, Hwang JY, et al. The risk of incident type 2 diabetes in a Korean metabolically healthy obese population: the role of systemic inflammation. The Journal of Clinical Endocrinology \& Metabolism. 2015;100(3):934-41.

71. Fan J, Song Y, Chen Y, Hui R, Zhang W. Combined effect of obesity and cardio-metabolic abnormality on the risk of cardiovascular disease: a meta-analysis of prospective cohort studies. International journal of cardiology. 2013;168(5):4761-8.

72. Kramer CK, Zinman B, Retnakaran R. Are metabolically healthy overweight and obesity benign conditions?: A systematic review and meta-analysis. Annals of Internal Medicine.

2013;159(11):758-69.

73. Meigs JB, Wilson PWF, Fox CS, Vasan RS, Nathan DM, Sullivan LM, et al. Body mass index, metabolic syndrome, and risk of type 2 diabetes or cardiovascular disease. J Clin Endocr Metab. 2006;91(8):2906-12.

74. Arnlov J, Ingelsson E, Sundstrom J, Lind L. Impact of body mass index and the metabolic syndrome on the risk of cardiovascular disease and death in middle-aged men. Circulation. 2010;121(2):230-6. Epub 2009/12/30.

75. Ogorodnikova AD, Kim M, McGinn AP, Muntner P, Khan U, Wildman RP. Incident cardiovascular disease events in metabolically benign obese individuals. Obesity (Silver Spring). 2012;20(3):651-9. Epub 2011/07/30.

76. Song Y, Manson JE, Meigs JB, Ridker PM, Buring JE, Liu S. Comparison of usefulness of body mass index versus metabolic risk factors in predicting 10 -year risk of cardiovascular events in women. The American journal of cardiology. 2007;100(11):1654-8.

77. Chang Y, Ryu S, Choi Y, Zhang Y, Cho J, Kwon M-J, et al. Metabolically Healthy Obesity and Development of Chronic Kidney Disease. Annals of Internal Medicine. 2016;164(5):305-12. 
78. Jung $\mathrm{CH}$, Lee MJ, Kang YM, Hwang JY, Kim EH, Park J-Y, et al. The risk of chronic kidney disease in a metabolically healthy obese population. Kidney international. 2015;88(4):843-50. 79. Panwar B, Hanks LJ, Tanner RM, Muntner P, Kramer H, McClellan WM, et al. Obesity, metabolic health, and the risk of end-stage renal disease. Kidney international. 2015;87(6):1216-22. 80. Renehan AG, Tyson M, Egger M, Heller RF, Zwahlen M. Body-mass index and incidence of cancer: a systematic review and meta-analysis of prospective observational studies. The Lancet. 2008;371(9612):569-78.

81. Bhaskaran K, Douglas I, Forbes H, dos-Santos-Silva I, Leon DA, Smeeth L. Body-mass index and risk of 22 specific cancers: a population-based cohort study of $5 \cdot 24$ million UK adults. The Lancet. 2014;384(9945):755-65.

82. Jokela M, Hamer M, Singh-Manoux A, Batty G, Kivimäki M. Association of metabolically healthy obesity with depressive symptoms: pooled analysis of eight studies. Mol Psychiatr. 2014;19(8):910-4.

83. Bauman AE, Sallis JF, Dzewaltowski DA, Owen N. Toward a better understanding of the influences on physical activity: the role of determinants, correlates, causal variables, mediators, moderators, and confounders. American journal of preventive medicine. 2002;23(2):5-14.

84. Cuthbertson DJ, Bell JA, Ng S, Kemp GJ, Kivimaki M, Hamer M. Dynapenic obesity and the risk of incident Type 2 diabetes: the English Longitudinal Study of Ageing. Diabetic Medicine. 2015. 85. Cleasby ME, Jamieson P, Atherton PJ. Insulin resistance and sarcopenia: mechanistic links between common co-morbidities. Journal of Endocrinology. 2016:JOE-15-0533.

86. Fukui M. Maintaining Metabolically Healthy Over-weight and Risk of Incident Type 2 Diabetes or Cardiovascular Disease. Int J Diabetol Vasc Dis Res S. 2015;3:1-6.

87. Arnlov J, Sundstrom J, Ingelsson E, Lind L. Impact of BMI and the metabolic syndrome on the risk of diabetes in middle-aged men. Diabetes Care.34(1):61-5.

88. Kim C-H, Kim H-K, Bae S-J, Kim E-H, Park J-Y. Independent impact of body mass index and metabolic syndrome on the risk of type 2 diabetes in Koreans. Metabolic Syndrome \& Related Disorders.10(5):321-5.

89. Hwang L-C, Bai C-H, Sun C-A, Chen C-J. Prevalence of metabolicallv healthy obesity and its impacts on incidences of hypertension, diabetes and the metabolic syndrome in Taiwan. Asia Pacific journal of clinical nutrition. 2012;21(2):227.

90. Kip KE, Marroquin OC, Kelley DE, Johnson BD, Kelsey SF, Shaw LJ, et al. Clinical importance of obesity versus the metabolic syndrome in cardiovascular risk in women a report from the Women's Ischemia Syndrome Evaluation (WISE) study. Circulation. 2004;109(6):706-13.

91. Ekelund $U$, Brage S, Besson H, Sharp S, Wareham NJ. Time spent being sedentary and weight gain in healthy adults: reverse or bidirectional causality? The American journal of clinical nutrition. 2008;88(3):612-7.

92. Hjorth MF, Chaput J-P, Ritz C, Dalskov S-M, Andersen R, Astrup A, et al. Fatness predicts decreased physical activity and increased sedentary time, but not vice versa: support from a longitudinal study in 8-to 11-year-old children. Int J Obesity. 2014;38(7):959-65.

93. Richmond RC, Smith GD, Ness AR, den Hoed M, McMahon G, Timpson NJ. Assessing causality in the association between child adiposity and physical activity levels: a Mendelian randomization analysis. PLoS Med. 2014;11(3):e1001618.

94. Smith GD, Ebrahim S. 'Mendelian randomization': can genetic epidemiology contribute to understanding environmental determinants of disease? International journal of epidemiology. 2003;32(1):1-22.

95. Evans DM, Davey Smith G. Mendelian randomization: new applications in the coming age of hypothesis-free causality. Annual review of genomics and human genetics. 2015;16:327-50.

96. Rana JS, Li TY, Manson JE, Hu FB. Adiposity compared with physical inactivity and risk of type 2 diabetes in women. Diabetes Care. 2007;30(1):53-8.

97. Weinstein AR, Sesso HD, Lee IM, Cook NR, Manson JE, Buring JE, et al. Relationship of physical activity vs body mass index with type 2 diabetes in women. JAMA. 2004;292(10):1188-94. 
98. Lee D-c, Sui X, Church TS, Lee I-M, Blair SN. Associations of cardiorespiratory fitness and obesity with risks of impaired fasting glucose and type 2 diabetes in men. Diabetes Care.

2009;32(2):257-62.

99. Fogelholm M. Physical activity, fitness and fatness: relations to mortality, morbidity and disease risk factors. A systematic review. Obesity Reviews. 2010;11(3):202-21.

100. Hu G, Tuomilehto J, Silventoinen K, Barengo N, Jousilahti P. Joint effects of physical activity, body mass index, waist circumference and waist-to-hip ratio with the risk of cardiovascular disease among middle-aged Finnish men and women. European heart journal. 2004;25(24):2212-9.

101. Weinstein AR, Sesso HD, Lee I-M, Rexrode KM, Cook NR, Manson JE, et al. The joint effects of physical activity and body mass index on coronary heart disease risk in women. Arch Intern Med. 2008;168(8):884-90.

102. Li TY, Rana JS, Manson JE, Willett WC, Stampfer MJ, Colditz GA, et al. Obesity as compared with physical activity in predicting risk of coronary heart disease in women. Circulation.

2006;113(4):499-506.

103. Jensen MK, Chiuve SE, Rimm EB, Dethlefsen C, Tjønneland A, Joensen AM, et al. Obesity, behavioral lifestyle factors, and risk of acute coronary events. Circulation. 2008;117(24):3062-9.

104. Janiszewski PM, Ross R. Physical activity in the treatment of obesity: beyond body weight reduction. Applied Physiology, Nutrition, and Metabolism. 2007;32(3):512-22.

105. Ross R, Dagnone D, Jones PJ, Smith H, Paddags A, Hudson R, et al. Reduction in obesity and related comorbid conditions after diet-induced weight loss or exercise-induced weight loss in men: a randomized, controlled trial. Annals of Internal Medicine. 2000;133(2):92-103.

106. Ross R, Hudson R, Stotz PJ, Lam M. Effects of exercise amount and intensity on abdominal obesity and glucose tolerance in obese adults: a randomized trial. Annals of Internal Medicine. 2015;162(5):325-34.

107. Ekkekakis P, Vazou S, Bixby W, Georgiadis E. The mysterious case of the public health guideline that is (almost) entirely ignored: call for a research agenda on the causes of the extreme avoidance of physical activity in obesity. Obesity Reviews. 2016.

108. Holmes MV, Lange LA, Palmer T, Lanktree MB, North KE, Almoguera B, et al. Causal effects of body mass index on cardiometabolic traits and events: a Mendelian randomization analysis. The American Journal of Human Genetics. 2014;94(2):198-208.

109. Würtz P, Wang Q, Kangas AJ, Richmond RC, Skarp J, Tiainen M, et al. Metabolic signatures of adiposity in young adults: Mendelian randomization analysis and effects of weight change. PLoS Med. 2014;11(12):e1001765.

110. Magkos F, Fraterrigo G, Yoshino J, Luecking C, Kirbach K, Kelly SC, et al. Effects of Moderate and Subsequent Progressive Weight Loss on Metabolic Function and Adipose Tissue Biology in Humans with Obesity. Cell metabolism. 2016.

111. Shin MJ, Hyun YJ, Kim OY, Kim JY, Jang Y, Lee JH. Weight loss effect on inflammation and LDL oxidation in metabolically healthy but obese $(\mathrm{MHO})$ individuals: low inflammation and LDL oxidation in MHO women. Int J Obesity. 2006;30(10):1529-34.

112. Karelis AD, Messier V, Brochu M, Rabasa-Lhoret R. Metabolically healthy but obese women: effect of an energy-restricted diet. Diabetologia. 2008;51(9):1752-4.

113. Arsenault BJ, Côté M, Cartier A, Lemieux I, Després J-P, Ross R, et al. Effect of exercise training on cardiometabolic risk markers among sedentary, but metabolically healthy overweight or obese postmenopausal women with elevated blood pressure. Atherosclerosis. 2009;207(2):530.

114. Janiszewski PM, Ross R. Effects of Weight Loss Among Metabolically Healthy Obese Men and Women. Diabetes Care. 2010;33(9):1957-9.

115. Kantartzis K, Machann J, Schick F, Rittig K, Machicao F, Fritsche A, et al. Effects of a lifestyle intervention in metabolically benign and malign obesity. Diabetologia. 2011;54(4):864-8. 


\section{Reference annotations}

5 and $6 *$ - Large high quality studies examining body mass index and mortality risk based on individual-level data

11 * - Comprehensive review on risk of incident diseases associated with obesity

12 * - Meta-analysis comparing the magnitude of effects of general and abdominal obesity indicators for incident type 2 diabetes

$20 * *$ - Study based on US NHANES data used to quote the prevalence of healthy obesity in the general population

$25 *$ - Extensive review on the physiological differences between subcutaneous and visceral fat tissue

28 and $29 *$ - Intervention studies proposing the adipose tissue expandability hypothesis

$35 *$ - Review of healthy obesity focusing on implications for clinical practice

37 * - Intervention study showing different metabolic effects of weight gain among initially healthy and unhealthy obese adults, reinforcing the adipose tissue expandability hypothesis

$38 *$ - Theoretical review of the protective effects of muscle contraction against fat tissue

54 * - Study comparing objectively assessed physical activity levels across metabolic and obesity groups

$59 * *$ - Study examining progressions from healthy to unhealthy obesity over 20 years

$64^{* *}$ - Meta-analysis of healthy obesity and incident type 2 diabetes 
$69 * *$ - Study examining the modifying effect of liver fat in risk of type 2 diabetes among healthy obese adults

$71^{* *}$ - Meta-analysis of healthy obesity and risk of incident cardiovascular disease and mortality as separate outcomes

$83 *$ - Explanation of physical activity as a confounder, mediator, or effect modifier

$93^{* *}$ - Study examining the causal association between genetically elevated body mass index and subsequent physical activity

$95 *$ - Recent review of the rationale for using genetic variants as proxies for modifiable risk factors to examine causality

108 and $109^{* *}$ - Studies examining causal associations between genetically elevated body mass index and metabolic risk factors 


\section{Duality of Interest}

The authors declare that there is no duality of interest associated with this manuscript.

\section{Funding}

None 
Figure 1 Proportion of obese adults who are considered healthy based on common and strict definitions of metabolic health

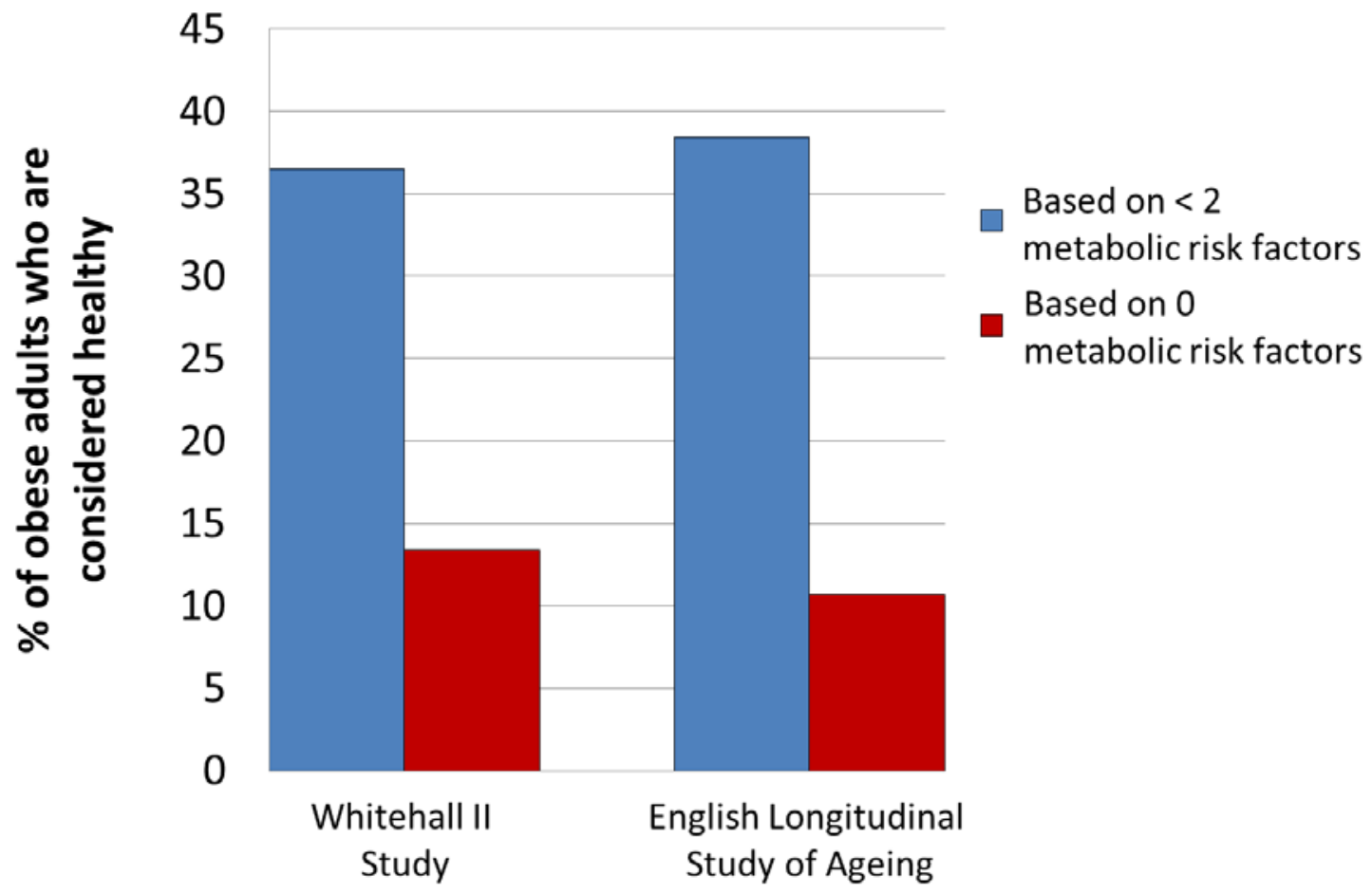

Legend Data for Whitehall II Study are from Bell et al., 2015, J Am Coll Cardiol and Bell et al., 2015

(2), J Am Coll Cardiol; Data for English Longitudinal Study of Ageing are from Hamer et al., 2015, Eur J Endocrinol 
Figure 2 Progressions to unhealthy obesity over 2 decades among non-obese and obese adults who are initially healthy

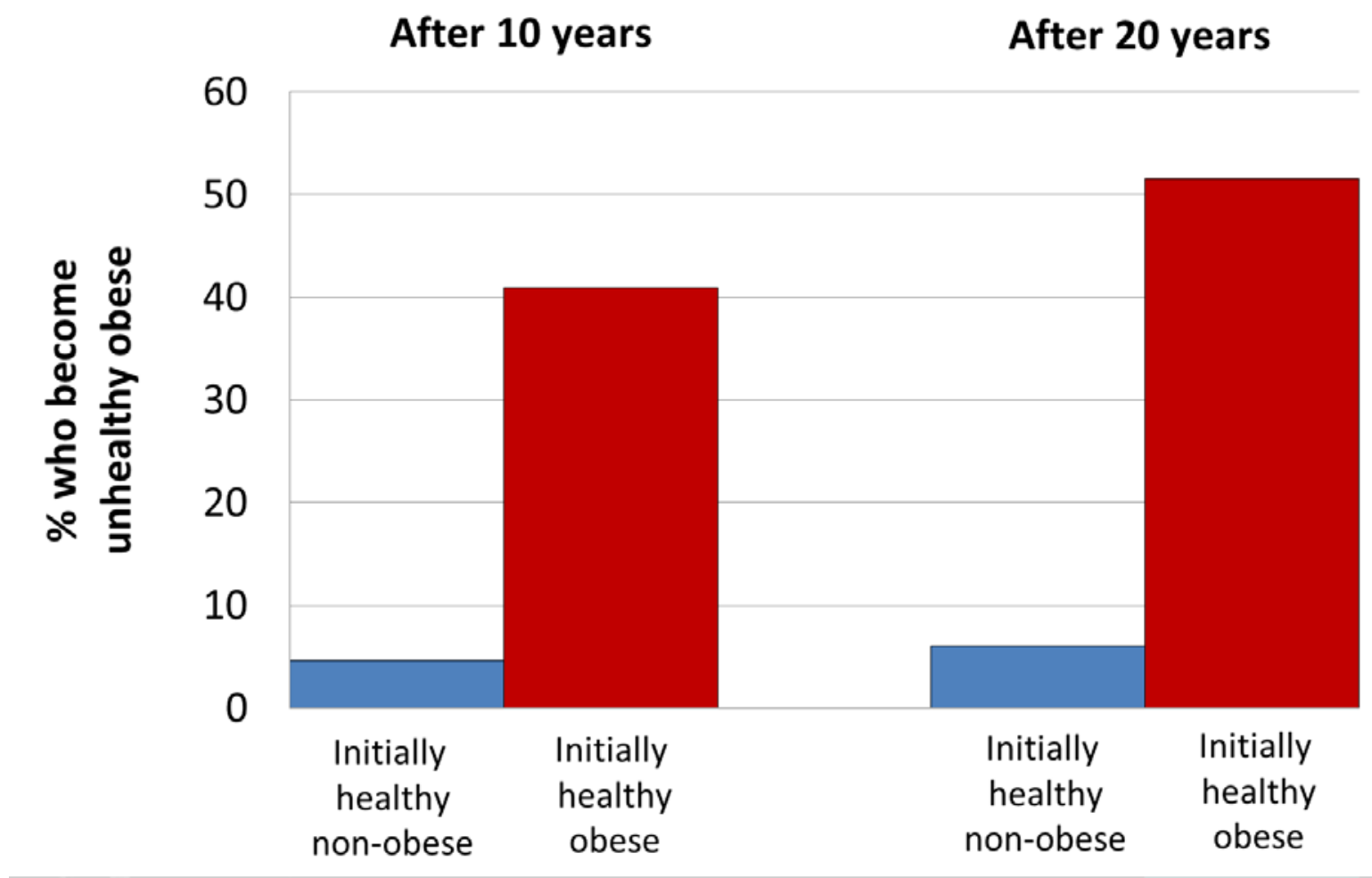

Legend Data are from Bell et al., 2015, J Am Coll Cardiol, based on 1677 initially healthy non-obese adults and 66 initially healthy obese adults followed-up over 20 years 
Figure 3 Clinical outcomes of healthy obesity established through meta-analyses

\section{Incident type 2 diabetes \\ Healthy normal-weight (ref) \\ Healthy obese \\ Unhealthy obese}

\section{Incident cardiovascular disease}

Healthy normal-weight (ref)

Healthy obese

Unhealthy obese

\section{Death from all causes}

Healthy normal-weight (ref)

Healthy obese

Unhealthy obese

\section{Death from cardiovascular causes}

Healthy normal-weight (ref)

Healthy obese

Unhealthy obese

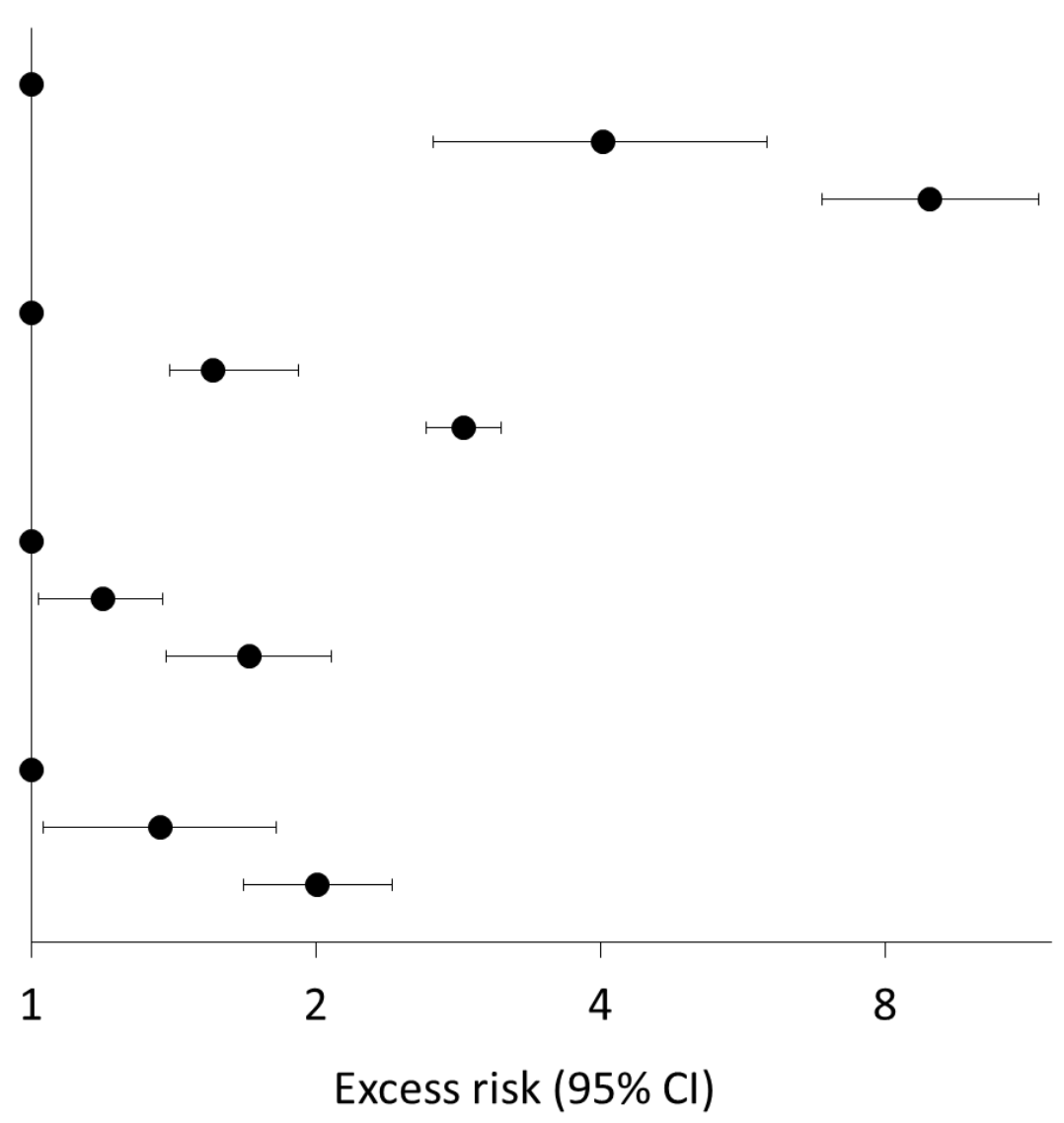

Excess risk $(95 \% \mathrm{Cl})$

Data are summary relative risks from meta-analyses which are multivariable-adjusted when possible; incident type 2 diabetes based on Bell et al. 2014,

Obes Rev; incident cardiovascular disease, death from all causes and death from cardiovascular causes based on Fan et al. 2013, Int J Cardiol. 